\title{
Does degree of alteration in effort sense caused by eccentric exercise significantly affect initial exercise hyperpnea in humans?
}

\author{
Norio Hotta ${ }^{1 *} \mathbb{D}$, Kaoru Yamamoto ${ }^{2}$, Hisayoshi Ogata', Patrick Maher ${ }^{3}$, Naoya Okumura $^{4}$ and Koji Ishida ${ }^{5}$
}

\begin{abstract}
Previous research has shown an exaggeration in exercise hyperpnea 2 days after eccentric exercise (ECC). Enhancement in central command has been suggested as one candidate to account for this effect given that ECC-induced neuromuscular dysfunction increases relative exercise intensity, thus resulting in reinforcement of effort sense. The purpose of this study was, therefore, to elucidate whether the degree of alteration in effort sense caused by ECC affects exercise hyperpnea. Ten subjects performed 20-s single-arm extension-flexion exercises with weight strapped to the wrist, and ventilatory response was measured before (Pre) and 2 days after ECC (D2). Relative exercise intensity at Pre was $5 \%$ of maximal voluntary contraction (MVC) of Pre, whereas that at D2 was $9 \%$ MVC of D2 because of decline in muscle strength. Ventilatory responses were significantly exaggerated at D2 with a significant increase in effort sense. Although effort sense was significantly reduced during exercise at D2 when wrist weight was subtracted to match relative exercise intensity at Pre (5\% MVC of D2), ventilatory responses were still significantly higher than those of Pre. After the disappearance of post-ECC muscle damage, subjects performed the same exercise with weight added (9\% MVC of Pre) so that effort was equalized to match that of D2; however, no significant increase in ventilatory response was detected. The fact that the extent of change in effort sense caused by ECC-induced neuromuscular dysfunction did not affect ventilatory response at the onset of exercise after ECC may suggest that the exaggeration of ventilatory response after ECC is caused by mechanisms other than alteration of the central command.
\end{abstract}

Keywords: Central command, Ventilation, Neuromuscular dysfunction, Delayed onset muscle soreness, Exercise onset

Abbreviations: ECC, Eccentric muscular work or eccentric exercise; DOMS, Delayed onset muscle soreness; Pre, Before eccentric muscular work or eccentric exercise; D2, 2 days after eccentric muscular work or eccentric

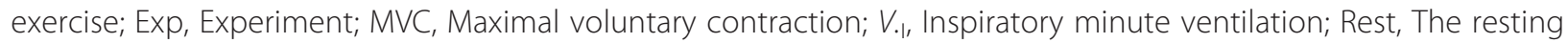
value of inspiratory minute ventilation; EX, The exercising value of inspiratory minute ventilation; RPE, Ratings of perceived exertion; VAS, Visual analog scale; EMG, Electromyogram

\section{Introduction}

Regulation of ventilatory response to physical activity is assumed to be controlled by neural and/or humoral mechanisms [1]. The neural controls are thought to consist of a "central command" [2] and afferent feedback signals via thin muscle afferents from exercising muscles [3]. Regarding central command, Bell [4] and

\footnotetext{
* Correspondence: horinori@isc.chubu.ac.jp

${ }^{1}$ College of Life and Health Sciences, Chubu University, Kasugai, Aichi

487-8501, Japan

Full list of author information is available at the end of the article
}

Ward [5] explain in their reviews that it is one of the central neural mechanisms by which the central motor commands to contracting muscles provide a parallel stimulus to the brainstem respiratory integrating areas.

Exercise consisting of eccentric muscular work (ECC), in which muscle fibers contract while being stretched, is well known to induce loss of muscle strength and/or mechanical hyperalgesia or delayed onset muscle soreness (DOMS) [6]. The tendency for an exaggerated ventilatory response to exercise 2 days after ECC has been demonstrated by several studies [7-11]. Although the mechanisms underlying this 
ventilatory response have not been fully elucidated, prior research points to an enhancement in the central command as a candidate to account for this phenomenon $[7,9,10]$. The reason for this is because ECC-induced neuromuscular dysfunction increases relative exercise intensity, thus resulting in a reinforcement in central motor commands that subsequently increases effort sense [12], and many researchers presume that effort sense and central command might alter in parallel (e.g., $[13,14])$.

In the present study, we aimed to reveal the effects of ECC-induced increases in relative exercise intensity or effort sense on exercise hyperpnea. To eliminate the effects of metabolic factors (e.g., chemoreflex and muscle metaboreflex) on ventilatory response and focus on neural mechanisms of ventilatory control (e.g., central command), we observed ventilatory responses at the onset of exercise (phase I) in the same way as in previous studies $[15,16]$.

\section{Methods}

Eight men and two women [age 23.4 \pm 5.4 years (mean \pm $\mathrm{SD})$, height $168.4 \pm 7.0 \mathrm{~cm}$, body mass $66.5 \pm 8.6 \mathrm{~kg}]$ volunteered to take part in this study. All subjects provided written consent after being informed of the experimental protocol and potential risks involved in their participation. This study received the approval of the Human Research Committee of Research Center of Health, Physical Fitness and Sports of Nagoya University.

A single-arm flexion-extension exercise, the same as that used in our previous study [17], served as the basis for data collection and ventilatory response measurements. Briefly, subjects sat with a weight belt equivalent to $5 \%$ of their maximal voluntary isometric elbow flexion (MVC) strength strapped to their wrist (regular weight trial). MVC strength was measured while seated with the elbow at $90^{\circ}$ utilizing a load cell and a strain amplifier [17] or a muscle strength dynamometer. Each subject flexed and extended his/her elbow joint once per second for $20 \mathrm{~s}$.

Subjects visited the laboratory four times. First, preliminary testing was carried out to familiarize the subjects with experimental procedures. The second visit involved measurement of ventilatory response to exercise (Pre),

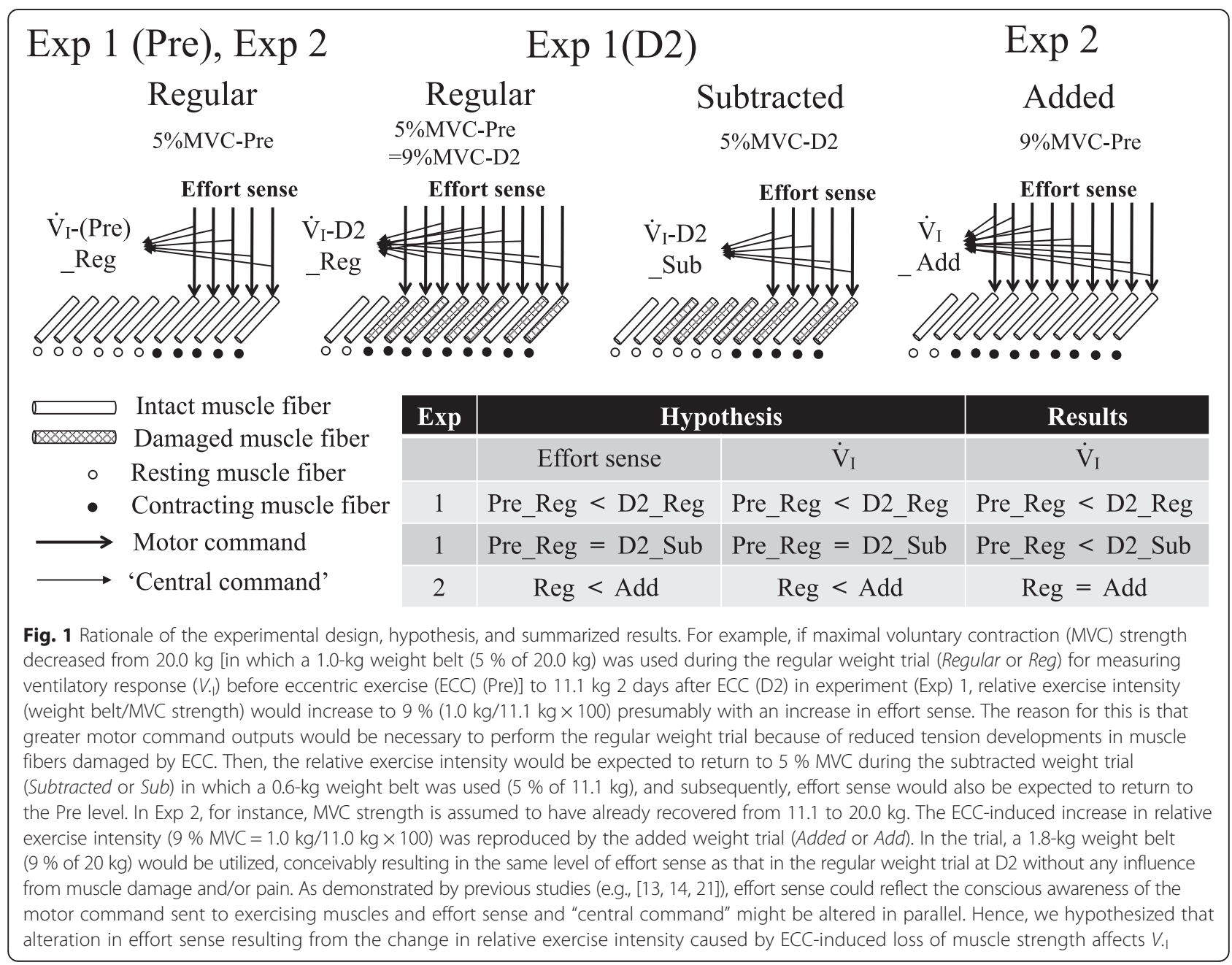


which was carried out just prior to subjects performing ECC in which the subjects completed three to six sets of 10 eccentric actions using a dumbbell set at $25-50 \%$ of MVC strength [17]. During the third visit, 2 days after ECC (D2), experiment (Exp) 1 was carried out to examine whether exaggerated ventilatory response changes if the relative exercise intensity resulting from ECC-induced muscle strength loss was "subtracted" to match the Pre intensity level (subtracted weight trial). Experiment 2 was performed during the final visit, after the effects of ECC would have disappeared, thus allowing for examination of whether ventilatory response alters under undamaged muscle state when relative exercise intensity is "added" to match the D2 level of ECC-induced muscle strength loss (added weight trial). The rationale of the experimental design and our hypothesis are summarized in Fig. 1.

Each subject repeated the exercise four to eight times. Breath-by-breath inspiratory minute ventilation $\left(V_{\text {.I }}\right)$ data were aligned with the onset of exercise and then linearly interpolated between each breath to yield data at 1-s intervals [16]. Afterwards, ensemble averaging was carried out. As in our previous study [17], we defined the average for $30 \mathrm{~s}$ before the start of exercise as the resting value of $V_{\text {.I }}$ (Rest) and the mean for the first $15 \mathrm{~s}$ of exercise as the exercising value of $V_{\text {. I }}(E X)$.

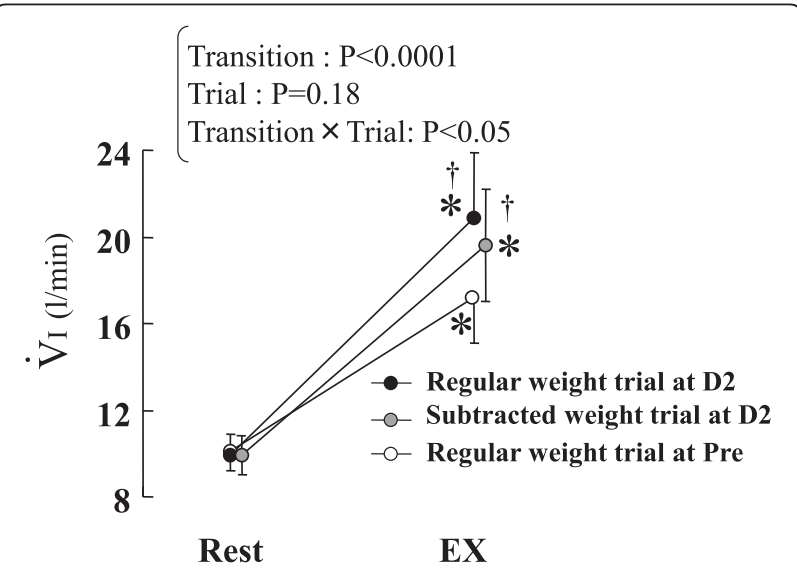

Fig. 2 Change in inspiratory minute ventilation $\left(V_{\text {.1 }}\right)$ from rest to the regular weight trials before (Pre) and 2 days after (D2) eccentric exercise (ECC) as well as the subtracted weight trial. The subtracted weight trial was performed at D2 and was arranged to compensate for the increase in exercise intensity attributed to ECC-induced loss of muscle strength during the regular weight trial at D2. The weight belts for the exercise were reduced so that the relative exercise intensity was equalized to that of the regular weight trial at Pre. The values of $V_{\text {. }}$ at Rest were the means for $30 \mathrm{~s}$ before the start of the trial. The values of $V_{\text {. }}$ at EX were the means for the first $15 \mathrm{~s}$ during each trial. Values are expressed as means $\pm \operatorname{SE}(n=10)$. Asterisks represent significant difference between Rest and EX $(P<0.05)$. Daggers represent significant difference compared to the regular weight trial at Pre $(P<0.05)$
A questionnaire for ratings of perceived exertion (RPE) to estimate the effort sense during exercise was used in previous studies (e.g., [13]). There was also a study that utilized RPE as a subjective index of central command [14]. We asked our subjects to indicate their RPE on the Borg scale [18] immediately after each exercise. We also evaluated the degree of muscle pain immediately after each exercise [17] using a visual analog scale (VAS) that had a 100-mm line with "no pain" on one end and "worst pain imaginable" on the other end [19]. The mean Borg scale ratings or VAS for each subject were then calculated and used as individually representative values.

A supplementary experiment was carried out for measurements in four additional healthy male volunteers $(21.0 \pm 1.4$ years, $170.8 \pm 6.1 \mathrm{~cm}$, and $69.5 \pm 9.9 \mathrm{~kg})$ to confirm whether central motor drive and/or motor unit recruitment during the 20-s single-arm flexion-extension exercise varied between before and after ECC. The subjects performed the exercise five times. An electromyogram (EMG) was obtained from elbow flexor muscles using an active electrode and the signals were amplified, filtered (high pass 15 and low pass $500 \mathrm{~Hz}$ ), and sampled at $1000 \mathrm{~Hz}$. The sampled signals were full-wave rectified, averaged by each of the 20 elbow flexion-extension signals that were measured by a goniometer, and normalized to the EMG obtained during the MVC [20]. Ensemble averaging was carried out across five repetitions, and then, a mean of 20 averaged rectified values during the exercise was used as an individually representative value. The electrode position was marked so that it could be placed in a nearly identical spot before and after ECC.

For statistical analysis, we performed multivariate ANOVAs with repeated measures. Factors included "experiment" (Exp 1 and 2), "transition" (Rest and EX), and "trial" (regular, subtracted, and added weight trials). Factors were chosen in order to allow for particular comparisons. If a significant $F$ value was observed in ANOVA, a Newman-Keuls post hoc test was used to identify specific differences. For paired-sample comparison, a paired $t$ test was used for data that were normally distributed and a Wilcoxon signed-rank test was used for data that were not normally distributed. Statistical analyses were carried out using StatView 5.0 and SPSS 14.0 software, and the level of significance was set at $5 \%$. All values are expressed as means $\pm \mathrm{SE}$.

\section{Results and discussion}

After ECC, MVC strength in all subjects declined from $27.1 \pm 2.5 \mathrm{~kg}$ at Pre to $15.4 \pm 1.5 \mathrm{~kg}$ at D2 $(P<0.0001)$. Additionally, muscle pain evaluations via a VAS showed that DOMS appeared in all subjects at D2 (from $0.0 \pm 0.0 \mathrm{~mm}$ at Pre to $54.5 \pm 8.0 \mathrm{~mm}$ at $\mathrm{D} 2, P=0.005$ ).

Data were collected during exercise sessions in which subjects' wrists were fitted with weight belts equivalent 


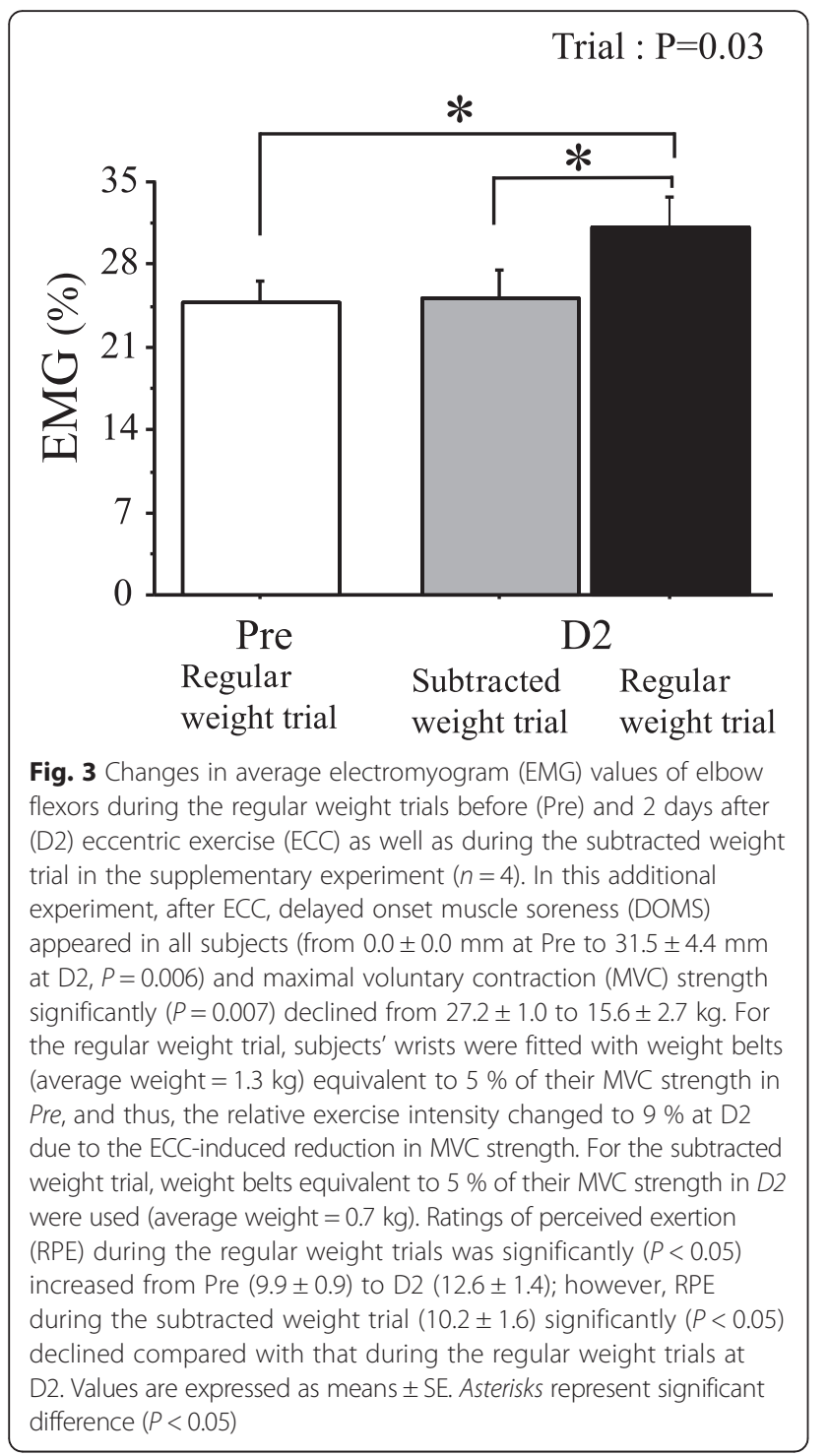

to $5 \%$ of their MVC strength at Pre (average weight = $1.4 \mathrm{~kg}$ ). Reflecting previous studies [7-11], a significant increase in $V_{\text {.I }}$ response was detected at D2 compared with that at Pre $(P<0.05)$ (Fig. 2); however, relative exercise intensity increased at D2 because the same weight belt used at Pre [average weight $=1.4 \mathrm{~kg}(9 \%$ MVC of D2)] was utilized despite the fact that the muscle strength declined. In fact, the EMG signal (Fig. 3) and RPE (from $11.1 \pm 0.7$ to $13.9 \pm 0.9$ ) at D2 during the exercise significantly $(P<0.05)$ increased compared with those at Pre, being in accordance with the results of prior studies $[7-9,20]$. It stands to reason that an enhancement in the central command might lie behind this phenomenon.

Immediately after initial data collection, the "subtracted weight trial" was arranged at D2. In that trial, exercise intensity was correlated to $5 \%$ of the MVC obtained at D2 by subtracting weight from the belt (average weight $=$ $0.8 \mathrm{~kg})$. This resulted in a significant $(P<0.05)$ decline in the EMG signal (Fig. 3) and RPE $(12.2 \pm 0.8)$ during the subtracted weight trial compared with that in the regular weight trial at D2. Nevertheless, $V_{\text {. I }}$ at EX was significantly $(P<0.05)$ higher than that in the regular weight trial at Pre, though no significant difference in $V_{\text {.I }}$ was detected between the subtracted weight trial and the regular weight trial at D2 (Fig. 2). Therefore, Exp 1 demonstrates that significant exaggeration in initial exercise hyperpnea occurred after ECC regardless of ECC-induced augmentations in relative exercise intensity or effort sense.

Shifting perspectives, to elucidate whether the extent of ECC-induced increases in relative exercise intensity and subsequent increases in effort sense per se affect initial exercise hyperpnea regardless of muscle state, Exp 2 was carried out under the condition in which the effects of ECC were absent. After the effects of ECC in Exp 1 had disappeared, subjects performed the following
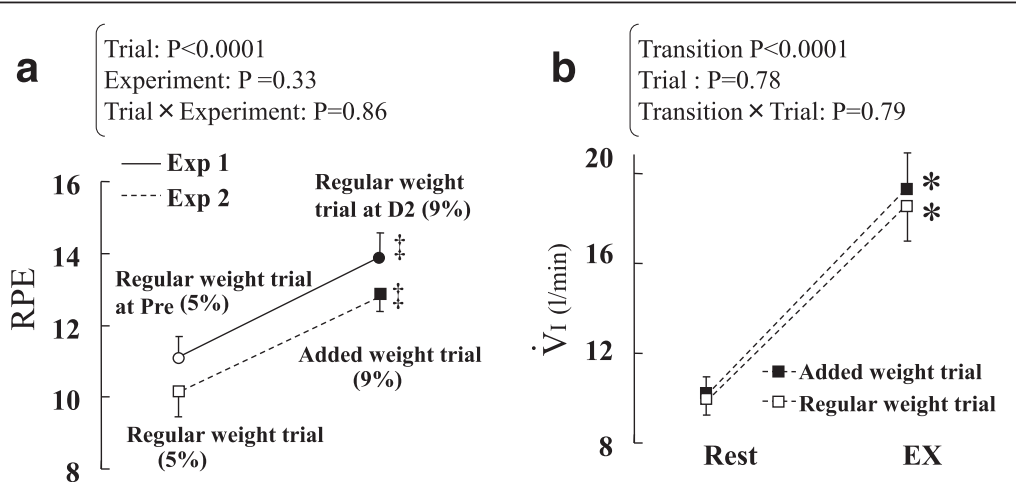

Fig. 4 a Change in ratings of perceived exertion (RPE) in the regular weight trials before (Pre) and 2 days after (D2) eccentric exercise (ECC) in experiment (Exp) 1 as well as from the regular weight trials to the added weight trial in Exp 2 performed after subjects had recovered from ECC-induced muscle damage. The added weight trial was arranged to simulate the increase in exercise intensity during the regular weight trials at D2. Double daggers represent significant difference between trials in each Exp $(P<0.05)$. Percentages in parentheses show the relative weight for exercise to maximal muscle strength. $\mathbf{b}$ Change in inspiratory minute ventilation $\left(V_{\text {. }}\right)$ from rest to the regular weight trials and the added weight trials. Abbreviations are the same as those in Fig. 2. Values are expressed as means \pm SE $(n=10)$ 
two types of exercise in random order. In exercise 1, each subject repeated the regular weight trial [average weight $=1.4 \mathrm{~kg}$ ( $5 \% \mathrm{MVC}$ of Pre)] as described earlier. In exercise 2, i.e., the "added weight trial," additional weight belts were bound to the subject's wrist to replicate an augmented relative intensity equal to that of the exercise at D2, in which MVC strength had decreased [average weight $=2.4 \mathrm{~kg}$ (average $9 \% \mathrm{MVC}$ of Pre; note that this value is the same as that of D2 in Exp 1)]. Figure 4a shows the changes in RPE. With no interaction between "trial" and "experiment," the added weight trial in Exp 2 should have simulated the change in effort sense seen during the exercise at D2 in Exp 1.

Figure $4 \mathrm{~b}$ shows the effects of the added weight trial on ventilatory response. There is no interaction between "trial" and "transition," implying that the added weight trial did not have any significant influence on ventilatory response at the onset of exercise. Hence, Exp 2 demonstrates that the degree of change in relative exercise intensity and effort sense influenced by ECC did not significantly affect the ventilatory response at the onset of exercise. The rationale and results of this study are summarized in Fig. 1.

\section{Conclusion}

Contrary to our hypothesis (Fig. 1), the degree of the alteration in effort sense resulting from the change in relative exercise intensity caused by ECC-induced loss of muscle strength did not significantly affect initial exercise hyperpnea. On the rationale that effort sense and central command might be altered in parallel (e.g., $[13,14])$, the results may suggest that post-ECC muscle condition changes the central command during exercise but that at least the extent of the change does not significantly relate to an exaggeration in ventilatory response at the onset of exercise after ECC.

\section{Acknowledgements}

We appreciate the cooperation of all subjects in the present study. We would also like to thank Dr. K. Watanabe for advising us.

\section{Authors' contributions}

$\mathrm{NH}$ contributed to all of the works. KY and NO contributed to the experiment and interpretation of the data. $\mathrm{HO}$ and PM contributed to the analysis and interpretation of the data and writing the paper. $\mathrm{KI}$ contributed to the management of possible risks and helped to draft the manuscript. All of the authors have read and approved the final manuscript.

\section{Competing interests}

The authors declare that they have no competing interests.

\section{Author details}

${ }^{1}$ College of Life and Health Sciences, Chubu University, Kasugai, Aichi 487-8501, Japan. ${ }^{2}$ Faculty of Human Health Sciences, Meio University, Nago, Okinawa, Japan. ${ }^{3}$ Morioka Junior College, Department of International Cultural Studies, Iwate Prefectural University, Takizawa, Iwate, Japan ${ }^{4}$ Graduate School of Life and Health Sciences, Chubu University, Kasugai, Aichi, Japan. ${ }^{5}$ Research Center of Health, Physical Fitness and Sports, Nagoya University, Nagoya, Aichi, Japan.
Received: 25 April 2016 Accepted: 4 August 2016

Published online: 24 August 2016

\section{References}

1. Ward SA. Ventilatory control in humans: constraints and limitations. Exp Physiol. 2007;92:357-66.

2. Paterson DJ. Defining the neurocircuitry of exercise hyperpnoea. J Physiol. 2014:592:433-44.

3. Kaufman MP. The exercise pressor reflex in animals. Exp Physiol. 2012;97:51-8.

4. Bell HJ. Respiratory control at exercise onset: an integrated systems perspective. Respir Physiol Neurobiol. 2006;152:1-15.

5. Ward SA. Control of the exercise hyperpnoea in humans: a modeling perspective. Respir Physiol. 2000;122:149-66.

6. Armstrong RB. Mechanisms of exercise-induced delayed onset muscular soreness: a brief review. Med Sci Sports Exerc. 1984;16:529-38.

7. Davies RC, Rowlands AV, Eston RG. Effect of exercise-induced muscle damage on ventilatory and perceived exertion responses to moderate and severe intensity cycle exercise. Eur J Appl Physiol. 2009;107:11-9.

8. Davies RC, Rowlands AV, Poole DC, Jones AM, Eston RG. Eccentric exerciseinduced muscle damage dissociates the lactate and gas exchange thresholds. J Sports Sci. 2011;29:181-9.

9. Gleeson M, Blannin AK, Zhu B, Brooks S, Cave R. Cardiorespiratory, hormonal and haematological responses to submaximal cycling performed 2 days after eccentric or concentric exercise bouts. J Sports Sci. 1995;13:471-9.

10. Hotta N, Sato K, Sun Z, Katayama K, Akima H, Kondo T, Ishida K. Ventilatory and circulatory responses at the onset of exercise after eccentric exercise. Eur J Appl Physiol. 2006;97:598-606.

11. Schneider DA, Berwick JP, Sabapathy S, Minahan CL. Delayed onset muscle soreness does not alter $\mathrm{O} 2$ uptake kinetics during heavy-intensity cycling in humans. Int J Sports Med. 2007;28:550-6.

12. Carson RG, Riek S, Shahbazpour N. Central and peripheral mediation of human force sensation following eccentric or concentric contractions. J Physiol. 2002;539:913-25.

13. Williamson JW, Fadel PJ, Mitchell JH. New insights into central cardiovascular control during exercise in humans: a central command update. Exp Physiol. 2006;91:51-8.

14. Victor RG, Pryor SL, Secher NH, Mitchell JH. Effects of partial neuromuscular blockade on sympathetic nerve responses to static exercise in humans. Circ Res. 1989:65:468-76.

15. Momen A, Bower D, Boehmer J, Kunselman AR, Leuenberger UA, Sinoway LI. Renal blood flow in heart failure patients during exercise. Am J Physiol Heart Circ Physiol. 2004;287:H2834-9.

16. Ishida K, Sato Y, Katayama K, Miyamura M. Initial ventilatory and circulatory responses to dynamic exercise are slowed in the elderly. J Appl Physiol. 2000;89:1771-7.

17. Hotta N, Yamamoto K, Katayama K, Ishida K. The respiratory response to passive and active arm movements is enhanced in delayed onset muscle soreness. Eur J Appl Physiol. 2009;105:483-91.

18. Borg GA. Psychophysical bases of perceived exertion. Med Sci Sports Exerc. 1982;14:377-81.

19. O'Connor PJ, Cook DB. Exercise and pain: the neurobiology, measurement, and laboratory study of pain in relation to exercise in humans. Exerc Sport Sci Rev. 1999;27:119-66.

20. Semmler JG, Ebert SA, Amarasena J. Eccentric muscle damage increases intermuscular coherence during a fatiguing isometric contraction. Acta Physiol (Oxf). 2013;208:362-75.

21. de Morree HM, Klein C, Marcora SM. Perception of effort reflects central motor command during movement execution. Psychophysiology. 2012:49:1242-53. 